\title{
Models of crustal heating in accreting neutron stars ${ }^{\star}$
}

\author{
P. Haensel and J. L. Zdunik
}

\author{
N. Copernicus Astronomical Center, Polish Academy of Sciences, Bartycka 18, 00-716 Warszawa, Poland \\ e-mail: [haensel;jlz]@camk.edu.pl
}

Received 30 August 2007 / Accepted 8 November 2007

\begin{abstract}
Aims. We calculate heating associated with non-equilibrium nuclear reactions in accreting neutron-star crusts, taking into account the suppression of neutrino losses demonstrated recently by Gupta et al. We consider two initial compositions of the nuclear-burning ashes, $A_{\mathrm{i}}=56$ and $A_{\mathrm{i}}=106$. We study the dependence of the integrated crustal heating on uncertainties plaguing pycnonuclear reaction models.

Methods. We use one-component plasma approximation, with compressible liquid-drop model of Mackie and Baym to describe nuclei. We follow the evolution of a crust shell from $10^{8} \mathrm{~g} \mathrm{~cm}^{-3}$ to $10^{13.6} \mathrm{~g} \mathrm{~cm}^{-3}$.

Results. The integrated heating in the outer crust agrees nicely with results of self-consistent multicomponent plasma simulations: earlier results fall between our curves obtained for $A_{\mathrm{i}}=56$ and $A_{\mathrm{i}}=106$. The total crustal heat per one accreted nucleon ranges between $1.5 \mathrm{MeV} /$ nucleon to $1.9 \mathrm{MeV} /$ nucleon for $A_{\mathrm{i}}=106$ and $A_{\mathrm{i}}=56$, respectively. The value of $Q_{\mathrm{tot}}$ weakly depends on the presence of pycnonuclear reactions at $10^{12}-10^{13} \mathrm{~g} \mathrm{~cm}^{-3}$. The remarkable insensitivity of $Q_{\mathrm{tot}}$ to the distribution of nuclear processes in accreted crust is explained.
\end{abstract}

Key words. dense matter - equation of state - stars: neutron - stars: general - X-rays: bursts - X-ray: binaries

\section{Introduction}

The neutron star crust that is not in full thermodynamic equilibrium constitutes a reservoir of energy, which can then be released during a star's evolution. The formation and structure of non-equilibrium neutron star crust has been considered by many authors (Vartanyan \& Ovakimova 1976; Bisnovatyi-Kogan \& Chechetkin 1979; Sato 1979; Haensel \& Zdunik 1990, 2003; Gupta et al. 2007). Such a state of the crust can be produced from the accretion of the matter onto a neutron star in a close low-mass X-ray binary, where the original crust built of a catalyzed matter could actually be replaced by a crust with a composition strongly deviating from the nuclear equilibrium one.

Heating due to non-equilibrium nuclear processes taking place in the outer and inner crusts of an accreting neutron star (deep crustal heating) was calculated by Haensel \& Zdunik (1990), who used a simple model of one-component plasma and assumed that the outer layers of the matter produced in the X-ray bursts consisted of pure ${ }^{56} \mathrm{Fe}$. Another simplification they used consisted in assuming the ground-state - ground-state nuclear transitions due to the electron captures. Consequently, they maximized neutrino losses. Their calculated total deep crustal heating, produced mainly in the inner crust, was $Q_{\text {tot }} \sim$ 1.4 MeV per one accreted nucleon. Haensel \& Zdunik (2003) recalculated deep crustal heating for different initial compositions of the outer layers and obtained similar values of $Q_{\text {tot }}=$ $1.2-1.4 \mathrm{MeV} /$ nucleon. Recently, heating of the outer crust of an accreting neutron star has been studied by Gupta et al. (2007), who went beyond the simple model of Haensel \& Zdunik (1990, 2003). Namely, Gupta et al. (2007) consider a multicomponent plasma, a reaction network of many nuclides, and included the contribution from the nuclear excited states. They find that

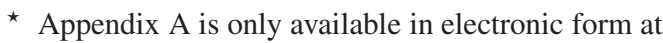
http://www . aanda.org electron captures in the outer crust lead mostly to the excited states of the daughter nuclei, which then deexcite heating the matter. Consequently, they find that the neutrino losses in the outer crust were negligible, which strongly increased the outer crust heating, compared to Haensel \& Zdunik (2003) (by a factor of four). However, the outer crust contributes only a small fraction of the $Q_{\text {tot }}$. Moreover, neutrino losses in the inner crust, where the bulk of $Q_{\text {tot }}$ is produced, are small. Therefore, the original value of Haensel \& Zdunik (1990), $Q_{\text {tot }} \sim 1.4 \mathrm{MeV} /$ nucleon, remains quite a reasonable estimate (see Sect. 4 of the present paper).

The phenomenon of deep crustal heating appears to be relevant for understanding the thermal radiation observed in the soft $\mathrm{X}$-ray transients (SXTs) in quiescence, when the accretion from a disk formed of plasma flowing from the low-mass companion star is switched off or strongly suppressed. Typically, the quiescent emission is much higher than the expected one for an old neutron star. It has been suggested that this is due to the interiors of neutron stars in SXTs being heated-up during relatively short periods of accretion and bursting by the non-equilibrium processes associated with nuclear reactions taking place in the deep layers of the crust (Brown et al. 1998). The deep crustal heating, combined with appropriate models of neutron-star atmosphere and interior, is used to explain observations of SXTs in quiescence. The luminosity in quiescence depends on the structure of the neutron-star core and particularly on the rate of neutrino cooling. This has opened a new possibility of exploring the internal structure and the equation of state of neutron stars via the confrontation of theoretical models with observations of quiescent SXTs (see Colpi et al. 2001; Rutledge et al. 2002; Yakovlev et al. 2003, 2004; Levenfish \& Haensel 2007).

Up to now, the crustal heating used in modeling SXTs was described using the model of Haensel \& Zdunik (1990, hereafter referred to as HZ90), then updated and generalized by 
Haensel \& Zdunik (2003, hereafter referred to as HZ03). In these models, the heat was produced during the active (accretion) episodes, when the outer layer of neutron star was sinking in the neutron star interior under the weight of accreted matter. The very outer layer was assumed to be composed of the ashes of the X-ray bursts in the active epoch. For simplicity, those ashes were assumed to be a one-component plasma $\left({ }^{56} \mathrm{Fe}\right.$ in HZ90 and ${ }^{56} \mathrm{Fe}$ and ${ }^{106} \mathrm{Pd}$ in HZ03). Under increasing pressure, the composition of a sinking matter element was changing in a sequence of nuclear reactions: electron capture, neutron emission and absorption, and finally also pycnonuclear fusion at densities exceeding $10^{12} \mathrm{~g} \mathrm{~cm}^{-3}$. As the nuclear processes were proceeding off-equilibrium, they were accompanied by the heat deposition in the crustal matter. The crustal heating was mostly supplied by the pycnonuclear fusion processes in the inner crust at $\rho=10^{12}-10^{13} \mathrm{~g} \mathrm{~cm}^{-3}$. This seemingly crucial rôle of pycnonuclear reactions is embarrassing, because their rates are plagued by huge uncertainties. As shown by Yakovlev et al. (2006), the uncertainty in the calculated rate of pycnonuclear fusion of two ${ }^{34} \mathrm{Ne}$ nuclei, first pycnonuclear fusion in the inner crust as predicted by the HZ90 model, can be as large as seven (!) orders of magnitude. Therefore, there is a basic uncertainty about which pycnonuclear fusions do occur and at what densities. Fortunately, as we show in the present paper, this uncertainty does not significantly affect the value of $Q_{\text {tot }}$. However, it implies an ignorance of the distribution of heat sources in the inner crust. Possible observational constraints on the distribution of heat sources in the inner crust are discussed in Sect. 6.

In the present paper we redo the calculations of the crustal heating taking uncertainties in the pycnonuclear reaction rates into account, as well as the suppression of the neutrino losses. We show that the uncertainties in the pycnonuclear reaction rates do not significantly affect the total heat release in the crust, while of course influencing the radial distribution of heat sources. We also show that the effect of the excited states of daughter nuclei, while very important in the outer crust, does not lead to a significant increase in the total crustal heating. Calculations are performed assuming two different initial nuclides produced in the X-ray bursts.

The plan of the paper is as follows. In Sect. 2 we briefly recall the scenario of the deep crustal heating, nuclear model used in simulations and describe the relevant physical processes acting in the crust of an accreting neutron star. Results from selected simulations of the nuclear evolution of a matter element compressed from $10^{8} \mathrm{~g} \mathrm{~cm}^{-3}$ to $10^{13.6} \mathrm{~g} \mathrm{~cm}^{-3}$ are presented in the form of figures in Sect. 3 and tables in the Appendix. Total crustal heating is calculated in Sect. 4 . In Sect. 5 we give an explanation of the weak model dependence of the total crustal heating (per one accreted nucleon). Our conclusions are presented in Sect. 6, where we also suggest an observational testing of the actual radial distribution of heat sources that could be helpful in putting constraints on the deep crustal heating models.

\section{Non-equilibrium nuclear processes}

\subsection{Outer crust}

In what follows we briefly describe the nuclear evolution scenario of HZ90 and HZ03, with a correction implied by the results of Gupta et al. (2007). Under the conditions prevailing in accreting neutron-star crust at $\rho>10^{8} \mathrm{~g} \mathrm{~cm}^{-3}$, matter is strongly degenerate and is relatively cold $\left(T<10^{8} \mathrm{~K}\right)$, so that thermonuclear processes involving charged particles can be assumed to be blocked by the Coulomb barrier. Consequently, for the densities lower than the threshold for the pycnonuclear fusion (which is very uncertain, see Yakovlev et al. 2006, $\rho_{\mathrm{pyc}} \sim$ $10^{12}-10^{13} \mathrm{~g} \mathrm{~cm}^{-3}$ ), the number of nuclei in an element of matter does not change during the compression resulting from the increasing weight of accreted matter. Let us recall, that we assume that only one nuclear species $(A, Z)$ is present at each pressure (one component plasma). Due to the nucleon pairing, stable nuclei in dense matter have even $N=A-Z$ and $Z$ (even-even nuclides). In the outer crust, in which free neutrons are absent, the electron captures, which proceed in two steps,

$$
\begin{aligned}
& (A, Z)+\mathrm{e}^{-} \longrightarrow(A, Z-1)+v_{\mathrm{e}}, \\
& (A, Z-1)+\mathrm{e}^{-} \longrightarrow(A, Z-2)+v_{\mathrm{e}}+Q_{j},
\end{aligned}
$$

lead to a systematic decrease in $Z$ (and increase in $N=A-Z$ ) with increasing density. The first capture in Eq. (1) proceeds in a quasi-equilibrium manner, with a negligible energy release. It produces an odd-odd nucleus, that is strongly unstable in dense medium and captures a second electron in an non-equilibrium manner, with energy release $Q_{j}$, where $j$ is the label of the nonequilibrium process.

\subsection{Outer crust: comparison of $H Z^{*}$ model with Gupta et al. (2007)}

In the original model of HZ90, electron captures were assumed to proceed from the ground state of the initial nucleus to the ground state of the daughter nucleus (GS-GS), and therefore most of the energy release was taken away by neutrinos (from $3 / 4$ to $5 / 6$ of $Q_{j}$, see Haensel \& Zdunik 2003, for a discussion of this point). Very recently, an extensive, multicomponent selfconsistent calculation of the nuclear evolution of an accreted matter element in the crust of a bursting neutron star was carried out by Gupta et al. (2007). As they have shown, electron captures to excited states (GS-ES) and subsequent de-excitation strongly reduce neutrino losses, so that nearly all released energy heats the crust matter. We therefore modified the original HZ model by neglecting the neutrino losses accompanying electron captures: this model will be denoted as HZ*. Consider a version $\mathrm{HZ}^{*}$ model, corresponding to a specific choice of the initial mass number $A_{\mathrm{i}}$. The integrated heat deposited in the crust in the layer with bottom density $\rho$ can then be obtained as

$$
Q(\rho)=\sum_{j\left(\rho_{j}<\rho\right)} Q_{j} .
$$

In Fig. 1 we compare $Q\left(\mu_{\mathrm{e}}\right)$, obtained for two HZ* models, with results of Gupta et al. (2007). We replaced $\rho$ variable by the electron Fermi energy $\mu_{\mathrm{e}}$ to facilitate the comparison. The curve of Gupta et al. (2007) lies between two extreme HZ* models; it has a very large number of small jumps. These two features reflect the multicomponent structure of crustal matter and large network of nuclear reactions in Gupta et al. (2007), with $50 \lesssim A_{\mathrm{i}} \lesssim 110$. Generally, viewing the simplicity of the HZ* model, its agreement with the multicomponent, self-consistent calculations of Gupta et al. (2007) is very good.

\subsection{Inner crust}

Above the neutron-drip point $\left(\rho>\rho_{\mathrm{ND}}\right)$, electron captures trigger neutron emissions,

$$
\begin{aligned}
& (A, Z)+\mathrm{e}^{-} \longrightarrow(A, Z-1)+v_{\mathrm{e}}, \\
& (A, Z-1)+\mathrm{e}^{-} \longrightarrow(A-\mathrm{k}, Z-2)+\mathrm{k} n+v_{\mathrm{e}}+Q_{j}
\end{aligned}
$$




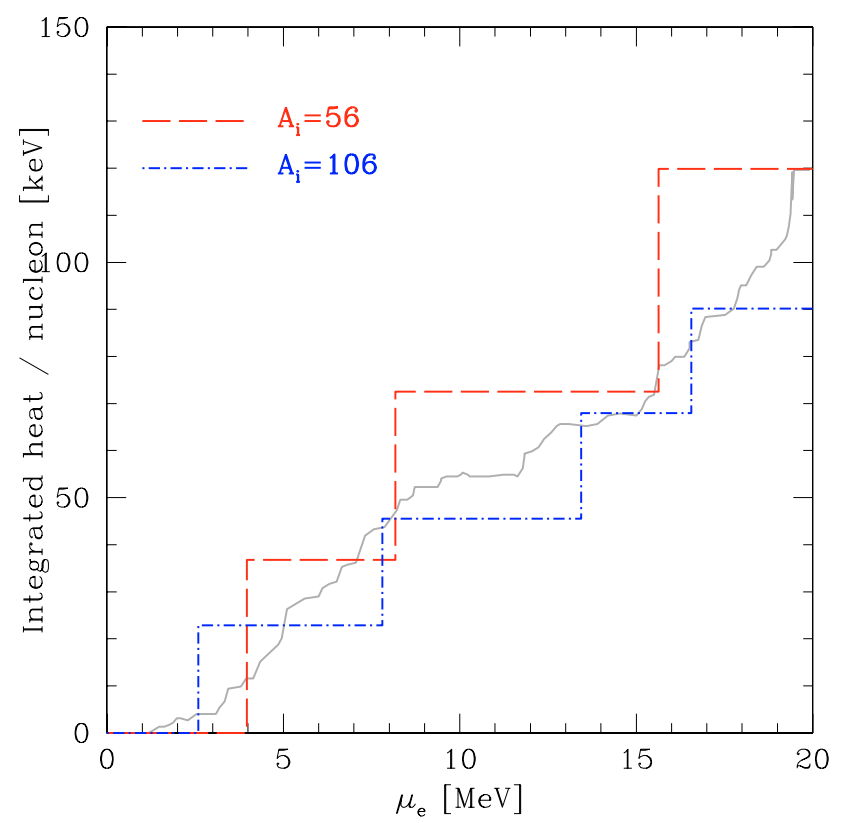

Fig. 1. (Color online) Integrated crustal heating per one accreted nucleon versus electron Fermi energy $\mu_{\mathrm{e}}$. The step-like curves were obtained using the $\mathrm{HZ}^{*}$ model of the present paper, for two choices of an initial nuclide. The continuous curve was obtained by Gupta et al. (2007).

Due to the electron captures, the value of $Z$ decreases with increasing density. In consequence, the Coulomb barrier prohibiting the nucleus-nucleus reaction lowers. This effect, combined with the decrease in the mean distance between the neighboring nuclei and a simultaneous increase of energy of the quantum zero-point vibrations around the nuclear lattice sites opens a possibility of the pycnonuclear reactions. The pycnonuclear fusion timescale $\tau_{\text {pyc }}$ is a very sensitive function of $Z$. The chain of the reactions (3) leads to an abrupt decrease in $\tau_{\text {pyc }}$ typically by 7 to 10 orders of magnitude. Pycnonuclear fusion switches on as soon as $\tau_{\text {pyc }}$ is smaller than the time of the travel of a piece of matter (due to the accretion) through the considered shell of mass $M_{\text {shell }}(N, Z), \tau_{\text {acc }} \equiv M_{\text {shell }} / \dot{M}$. The masses of the shells are on the order of $10^{-5} M_{\odot}$. As a result, in the inner crust the chain of reactions (3) in several cases is followed by the pycnonuclear reaction on a timescale much shorter than $\tau_{\text {acc }}$. Denoting $Z^{\prime}=Z-2$, we then have

$$
\begin{aligned}
& \left(A, Z^{\prime}\right)+\left(A, Z^{\prime}\right) \longrightarrow\left(2 A, 2 Z^{\prime}\right)+Q_{j, 1}, \\
& \left(2 A, 2 Z^{\prime}\right) \longrightarrow\left(2 A-\mathrm{k}^{\prime}, 2 Z^{\prime}\right)+\mathrm{k}^{\prime} n+Q_{j, 2}, \\
& \ldots \quad \ldots \quad \ldots \quad \ldots+Q_{j, 3},
\end{aligned}
$$

where "..." correspond to some unspecified chain of the electron captures accompanied by neutron emissions. The total heat deposition in matter, resulting from a chain of reactions involving a pycnonuclear fusion, is $Q_{j}=Q_{j, 1}+Q_{j, 2}+Q_{j, 3}$. In contrast to HZO3 and in accordance with Gupta et al. (2007), we neglect the neutrino losses accompanying the non equilibrium electron captures.

Our model of atomic nuclei is described in detail in HZ90. Using our nuclear-evolution code, we evolved an element of matter consisting initially of nuclei $\left(A_{i}, Z_{\mathrm{i}}\right)$ immersed in an electron gas, from $\rho_{\mathrm{i}}=10^{8} \mathrm{~g} \mathrm{~cm}^{-3}$ to $\rho=\rho_{\mathrm{f}}>5 \times 10^{13} \mathrm{~g} \mathrm{~cm}^{-3}$. Our results for the composition and crustal heating are presented in the next section and in the Appendix.
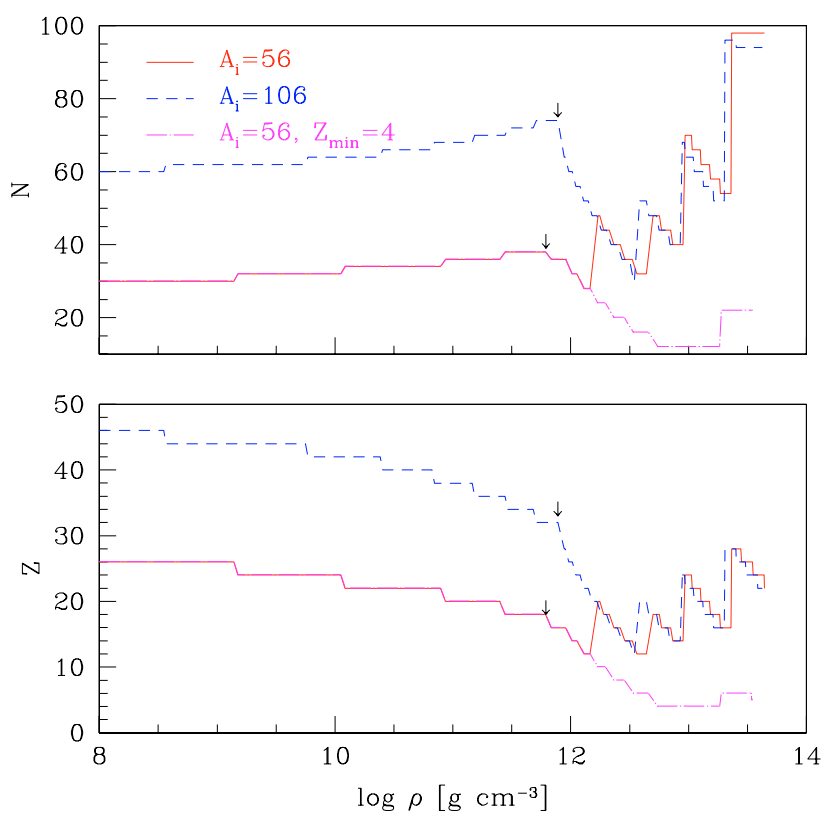

Fig. 2. (Color online) $Z$ and $N$ of nuclei, versus matter density in an accreting neutron-star crust, for different models of dense matter. Solid line: $A_{\mathrm{i}}=56$; dashed line: $A_{\mathrm{i}}=106$. Dash-dot line: continuation of $A_{\mathrm{i}}=$ 56 evolution but with pycnonuclear fusion blocked until $Z=Z_{\min }=4$. Each change of $N$ and $Z$, which takes place at a constant pressure, is accompanied by a jump in density (see HZ90 for detailed discussion of this point). Small steep segments connect the top and the bottom density of thin reaction shell. Arrows indicate positions of the neutron drip point.

\section{Results of simulations: reactions, heating, compositions}

The compositions of accreted neutron star crusts are shown in Fig. 2 and in tables in the Appendix. These results describe crusts built of accreted and processed matter up to the density $5 \times 10^{13} \mathrm{~g} \mathrm{~cm}^{-3}$. At a constant accretion rate $\dot{M}=\dot{M}_{-9} \times$ $10^{-9} M_{\odot} / \mathrm{yr}$, this will take $\sim 10^{6} \mathrm{yr} / \dot{M}_{-9}$. During that time, a shell of X-ray burst ashes will be compressed from $\sim 10^{8} \mathrm{~g} \mathrm{~cm}^{-3}$ to $\sim 10^{13} \mathrm{~g} \mathrm{~cm}^{-3}$.

Two different compositions of X-ray burst ashes at $\lesssim 10^{8} \mathrm{~g} \mathrm{~cm}^{-3}$ were assumed. In the first case, $A_{\mathrm{i}}=56, Z_{\mathrm{i}}=26$, like in HZ90. In the second scenario we followed HZ03, with $A_{\mathrm{i}}=106$, to imitate nuclear ashes obtained by Schatz et al. (2001). The value of $Z_{i}=46$ then stems from the condition of beta equilibrium at $\rho=10^{8} \mathrm{~g} \mathrm{~cm}^{-3}$. The density dependence of $Z$ and $N=A-Z$ within the accreted crust is shown in Fig. 2. After pycnonuclear fusion region had been reached, both curves converged (as explained in HZ03, this results from $A_{\mathrm{i}}$ and $Z_{\mathrm{i}}$ in two scenarios differing by a factor of about two). Suppressing pycnonuclear fusion in the $A_{\mathrm{i}}=56$ case until $Z=Z_{\min }=4$ yields the bottom (dash-dot) curve.

In Fig. 3 we show the heat deposited in the matter, per one accreted nucleon, in the thin shells in which non-equilibrium nuclear processes are taking place. Actually, reactions proceed at a constant pressure, and there is a density jump within a thin "reaction shell". The vertical lines whose height gives the heat deposited in matter are drawn at the density at the bottom of the reaction shell. One notices a specific dependence of the number of heat sources and the heating power of a single source on assumed $A_{\mathrm{i}}$. In the case of $A_{\mathrm{i}}=56$ the number of sources is smaller, 


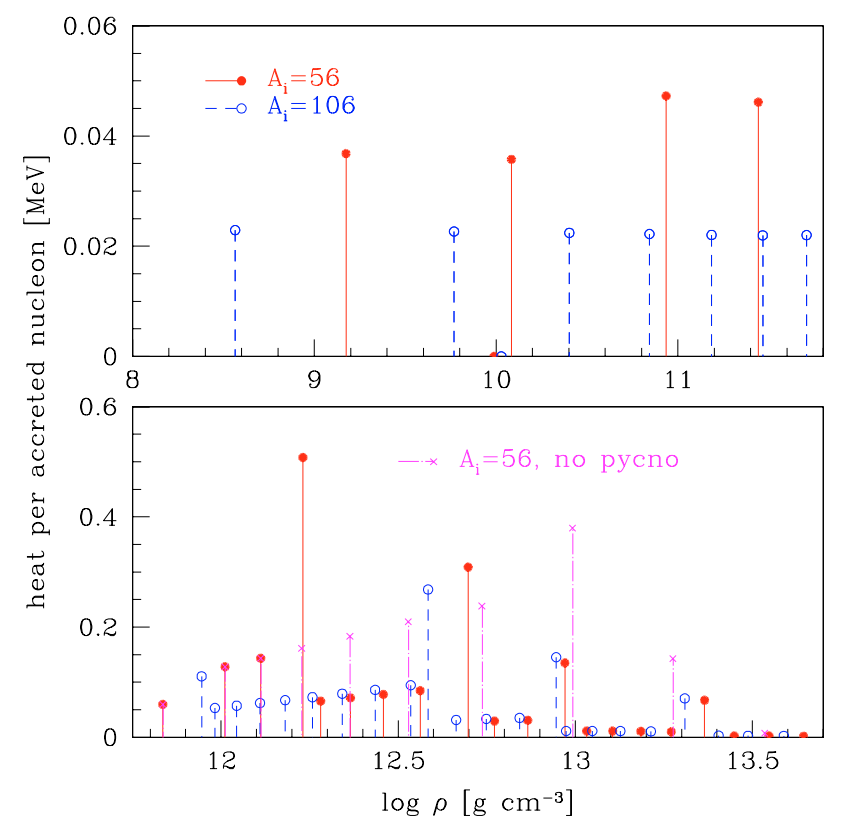

Fig. 3. (Color online) Heat sources in the outer (upper panel) and inner (lower panel) crust for three $\mathrm{HZ}^{*}$ models. Vertical lines, positioned at the density at the bottom of the reaction shell, represent the heat per one accreted nucleon. Labels as in Table 2.

and their heat-per-nucleon values $Q_{j}$ significantly larger, than for $A_{\mathrm{i}}=106$.

Results presented in Tables A.1-A.4 exhibit several characteristic features. One notices the importance of heating via neutron emission following immediately after quasi-equilibrium electron captures in the inner crust. There are also characteristic differences in the strength and number of heat sources per pressure interval. Let us consider two evolution tracks for $A_{\mathrm{i}}=56$ and $A_{\mathrm{i}}=106$, assuming that pycnonuclear fusion is suppressed until $Z=4$. In the process of neutronization, the heavier nucleus undergoes significantly more quasi-equilibrium electron captures and the heating at each source is much weaker than for the lighter initial nucleus. We may explain this systematic difference using the liquid drop model of nuclei. In the zeroth approximation, the Coulomb and surface contributions to the energy are neglected. This gives a so-called bulk approximation of the inner neutron star crust. Formally, this approximation corresponds to $A, Z \longrightarrow \infty$. Then, $Z / A$ in beta equilibrium changes continuously with increasing pressure. When approaching this limit, the number of heat sources tends to infinity but the heat release per one source tends to zero. Including Coulomb, surface, and other "finite size" components to the energy per nucleon makes $A$ and $Z$ finite. Thresholds appear for the electron captures and a certain number of discrete heat sources. Lower $A_{\mathrm{i}}$ corresponds to stronger "finite size" effects, higher electron capture thresholds, a smaller number of heat sources, and larger heat release at each source. With higher $A_{\mathrm{i}}$, one gets closer to the bulk limit, and therefore the heat releases per source is lower while the number of heat sources is greater.

\section{Integrated crustal heating}

The quantity $Q(\rho)$ for three specific models of compressional evolution is plotted in Fig. 4. In all three cases, we set $A_{\mathrm{i}}=56$ and $Z_{\mathrm{i}}=26$. For the first model, we neglect neutrino losses; its integrated heat is always the highest. The second model is used to visualize the importance of excited states of the daughter

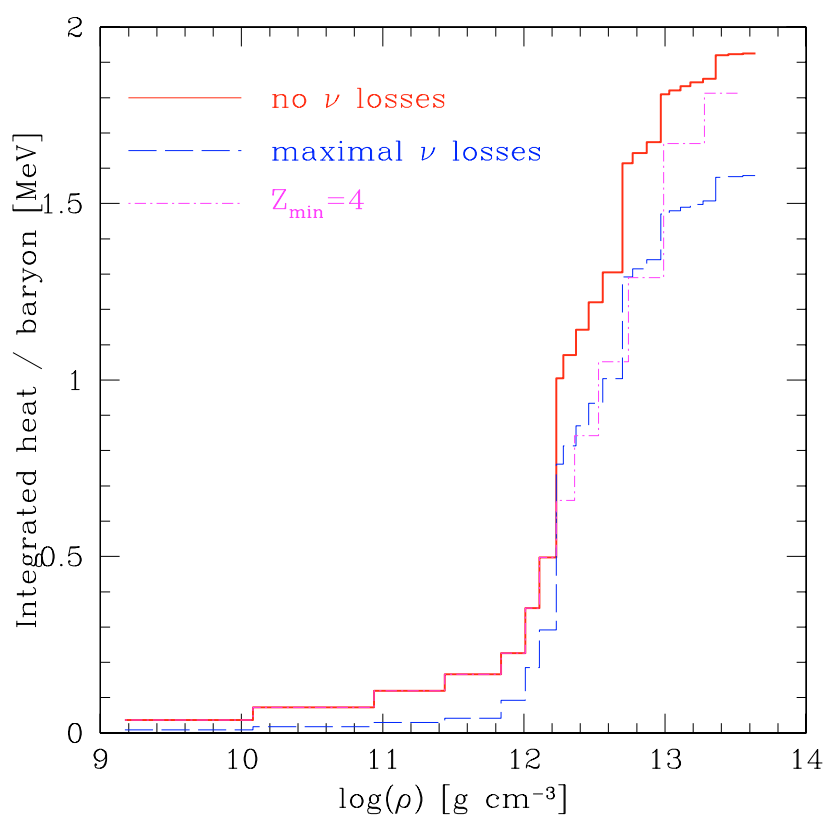

Fig. 4. (Color online) Integrated heat released in the crust, $Q(\rho)$ (per one accreted nucleon) versus $\rho$, assuming initial ashes of pure ${ }^{56} \mathrm{Fe}$. Solid line: HZ* model of the present paper, with suppressed neutrino losses. Long dashes: GS-GS transitions in electron captures, with maximal neutrino losses. Dash-dot line: No neutrino losses, with pycnonuclear fusion blocked until $Z=Z_{\min }=4$.

Table 1. Total crustal heating $Q_{\mathrm{tot}}$ for $A_{\mathrm{i}}=56$ and $A_{\mathrm{i}}=106$.

\begin{tabular}{ccc|c|c}
\hline \hline & $\begin{array}{c}\text { Model } \\
\text { pycno }\end{array}$ & $v$ losses & $\begin{array}{c}A_{\mathrm{i}}=56 \\
Q_{\text {tot }}\end{array}$ & $\begin{array}{c}A_{\mathrm{i}}=106 \\
Q_{\mathrm{tot}}\end{array}$ \\
\hline $\mathrm{HZ}^{* 1}$ & no blocking & none & $1.93 \mathrm{MeV}$ & $1.48 \mathrm{MeV}$ \\
$\mathrm{HZO3}^{2}$ & no blocking & maximal & $1.58 \mathrm{MeV}$ & $1.16 \mathrm{MeV}$ \\
$\mathrm{HZ}^{*}$ & $Z_{\min }=6$ & none & $1.93 \mathrm{MeV}$ & $1.44 \mathrm{MeV}$ \\
$\mathrm{HZ}^{*}$ & $Z_{\min }=4$ & none & $1.85 \mathrm{MeV}$ & $1.35 \mathrm{MeV}$ \\
\hline
\end{tabular}

${ }^{1}$ Present paper, ${ }^{2}$ Haensel \& Zdunik (2003). Third and fourth lines: results obtained when neutrino losses are suppressed and pycnonuclear fusion is blocked down to $Z_{\min }=6$ and $Z_{\min }=4$, respectively.

nuclei in the electron captures. For this model we assumed that the nuclear transitions associated with electron captures are of the GS-GS type, which maximizes the neutrino losses. While the effect is dramatic for $\rho \lesssim 10^{12} \mathrm{~g} \mathrm{~cm}^{-3}$, it only implies a $20 \%$ underestimate of $Q$ above $10^{12.5} \mathrm{~g} \mathrm{~cm}^{-3}$. But the most interesting is perhaps the effect of literally switching off the pycnonuclear reactions, assumed in the third scenario. This was done by assuming that the pycnonuclear fusion is blocked until the nuclear charge goes down to $Z_{\min }=4$. And yet, for $\rho_{\mathrm{b}}>10^{13} \mathrm{~g} \mathrm{~cm}^{-3}, Q$ is very similar to what is obtained in the first scenario, which was most advantageous as far as the crust heating was concerned. Namely, a missing pycnonuclear heating at $\rho \sim 10^{12} \mathrm{~g} \mathrm{~cm}^{-3}$ is efficiently compensated by the electron captures accompanied by neutron emission within the density decade $10^{12}-10^{13} \mathrm{~g} \mathrm{~cm}^{-3}$. The values of $Q$ saturate above $10^{13.6} \mathrm{~g} \mathrm{~cm}^{-3}$, where $80 \%$ of nucleons are in a neutron gas phase. All in all, for three scenarios with $A_{\mathrm{i}}=56$, the total deep crustal heat release is (1.6-1.9) MeV/nucleon. For $A_{\mathrm{i}}=106$, numbers are shifted downward by about $0.4 \mathrm{MeV} /$ nucleon. The summary of our results for the total heat release is given in Table 1. 


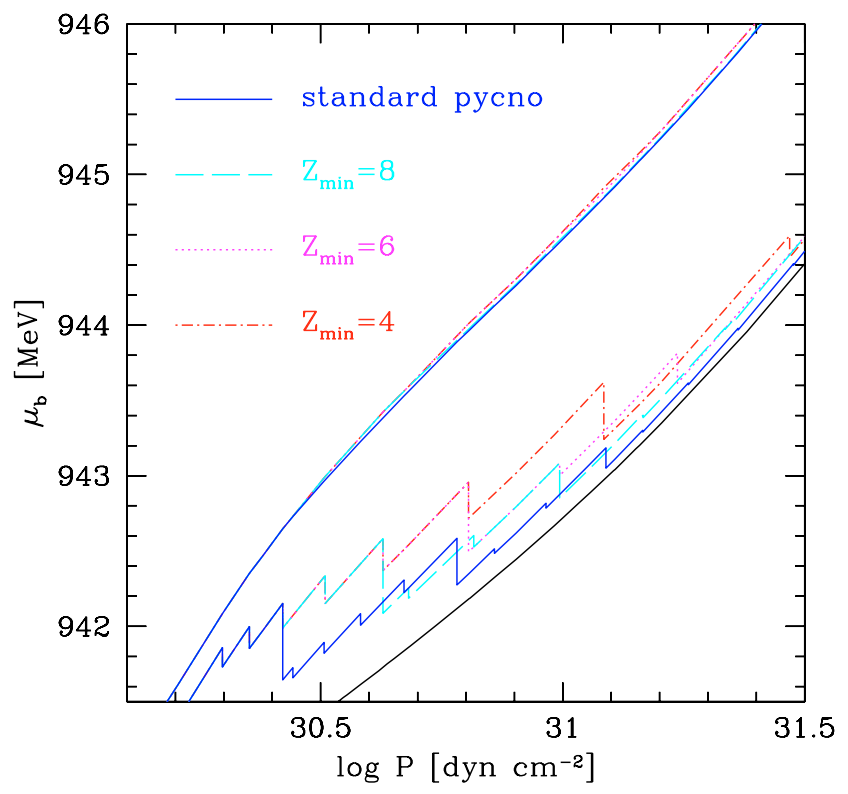

Fig. 5. (Color online) Baryon chemical potential $\mu_{\mathrm{b}}(P)$ for different versions of blocking of pycnonuclear fusion. "Standard pycno" corresponds to $\mathrm{HZ} *$ model of the present paper, with $A_{\mathrm{i}}=56$. Three other curves correspond to pycnonuclear fusion suppressed until to $Z=Z_{\min }=8,6,4$, respectively. The upper continuous curves, which nearly coincide, are defined as $\bar{\mu}_{\mathrm{b}}(P) \equiv \mu_{\mathrm{b}}(P)+\sum_{j\left(P_{j}<P\right)} Q_{j}$. Dependence of $\bar{\mu}_{\mathrm{b}}(P)$ on the evolutionary scenario is negligible. The lowest smooth solid curve - cold catalyzed matter.

\section{Constancy of the total heat release}

The remarkably weak dependence of the total heat release in the crust, $Q_{\mathrm{tot}}$, on the nuclear history of an element of matter undergoing compression from $\sim 10^{8} \mathrm{~g} \mathrm{~cm}^{-3}$ to $\sim 10^{13.6} \mathrm{~g} \mathrm{~cm}^{-3}$ deserves an explanation. Let us therefore study the most relevant thermodynamic quantity, the Gibbs free energy per nucleon, which is the baryon chemical potential, $\mu_{\mathrm{b}}$. In the $T=0$ approximation, $\mu_{\mathrm{b}}(P)=[\mathcal{E}(P)+P] / n_{\mathrm{b}}(P)$. Minimizing $\mu_{\mathrm{b}}(P)$ at a fixed $P$, with respect to the independent thermodynamical variables $\left(A, Z\right.$, mean free neutron density $\bar{n}_{n}$, mean baryon density $n_{\mathrm{b}}$, size of the Wigner-Seitz cell, etc.), under the constraint of electric charge neutrality, $\bar{n}_{\mathrm{p}}=\bar{n}_{\mathrm{e}}$, we get the the ground state of the crust a given $P$. This state is called "cold catalyzed" matter, and its baryon chemical $\mu_{\mathrm{b}}^{(0)}(P)$ is represented in Fig. 5 by a solid black line. All other $\mu_{\mathrm{b}}(P)$ curves, showed in Fig. 5, display discontinuous drops due to non-equilibrium reactions included in a given model, and lie above the solid black one. This visualizes that non-catalyzed matter is a reservoir of energy, released in non-equilibrium processes that pushes down matter closer to the absolute ground state. In spite of dramatic differences between different $\mu_{\mathrm{b}}(P)$ in the region where the bulk of non-equilibrium reactions and heating occur, $P=\left(10^{30}-10^{31.5}\right)$ dyn $\mathrm{cm}^{-2}$, these functions tend to the ground state one for $P \gtrsim 10^{32} \mathrm{dyn} \mathrm{cm}^{-2}$. The general structure of different $\mu_{\mathrm{b}}(P)$ is similar. At the same $P$, their continuous segments have nearly the same slope. What differs $\mu_{\mathrm{b}}(P)$ are discontinuous drops, by $Q_{j}$, at reaction thresholds $P_{j}$. The functions $\mu_{\mathrm{b}}(P)$ can therefore be expressed as

$\mu_{\mathrm{b}}(P) \approx \bar{\mu}_{\mathrm{b}}(P)-\sum_{j\left(P_{j}<P\right)} Q_{j}$,

where $\bar{\mu}_{\mathrm{b}}(P)$ is a smooth function of $P$, independent of the evolutionary track (for explanation, see Fig. 5 and its caption). For $P>10^{33.5} \mathrm{dyn} \mathrm{cm}^{-2}$, the values of $Q_{j}$ are negligibly low, and all $\mu_{\mathrm{b}}(P)$ become quite close to the ground state line. This implies that the sum $\sum_{j} Q_{j}$ has to be, to a rather good precision, independent of evolutionary track.

\section{Discussion and conclusion}

In the present paper we reconsidered a model of deep crustal heating, formulated originally in (Haensel \& Zdunik 1990, 2003). Following Gupta et al. (2007), we suppressed neutrino losses associated with electron captures. In this way we got the HZ* model of crustal heating that despite its simplicity (one component plasma) agrees nicely with results of self-consistent multi-component calculations of Gupta et al. (2007). Using the $\mathrm{HZ}^{*}$ model we obtained the total crustal heating $Q_{\mathrm{tot}}=1.5 \mathrm{MeV}$ and $1.9 \mathrm{MeV} /$ nucleon, for the initial ashes consisting of ${ }^{106} \mathrm{Pd}$ and ${ }^{56} \mathrm{Fe}$, respectively. We studied the dependence of crustal heating on the location of the pycnonuclear fusion processes within the crust. It turned out that the total crustal heating (per one accreted nucleon) is quite insensitive to the depth at which pycnonuclear fusion occurs, with $Q_{\text {tot }}$ varying at most by $0.2 \mathrm{MeV}$, and we presented an explanation of this feature.

Maximal neutrino losses, implied by the assumption of the ground-state - ground-state electron captures in Haensel \& Zdunik (1990, 2003), led to severe underestimations of the heating of the outer crust (Gupta et al. 2007). However, the outer crust gives a rather small contribution to the total crustal heating, $Q_{\text {tot }}$, and the underestimation of $Q_{\text {tot }}$ is less than $25 \%$. Composition of the initial ashes of the X-ray bursts can be more important for the value of $Q_{\mathrm{tot}}$, as shown already in Haensel \& Zdunik (2003).

The insensitivity of $Q_{\text {tot }}$ to the very uncertain rates of pycnonuclear fusion means that this tremendous uncertainty, unlikely to be removed in spite of future theoretical efforts, does not affect the thermally equilibrated quiescent state of the SXTs. This may seem good news. On the other hand, this means that the studies of SXTs in quiescence will not improve our knowledge of pycnonuclear fusion in dense plasma: this is the bad news.

Fortunately, the situation changes with the access to observations of the thermal relaxation in SXTs after the accretion episode. This phenomenon cannot be observed in standard SXTs that remain in accreting state for days to weeks. Thermal relaxation can be observed only in so-called persistent SXTs, characterized by accretion states lasting for years to decades. This thermal relaxation, called initial cooling, has been observed in KS 1731-260 and MXB 1659-29 (Cackett et al. 2006). Let us focus on KS 1731-260. After 12.5 years of accretion and associated crustal heating, the crust of KS 1731-260 has become significantly hotter than the neutron star core. After accretion stopped, the heat cumulated in the crust diffused over the star, and the stellar surface cooled. The cooling curve of KS 1731-260 toward the quiescent state has been obtained by Cackett et al. (2006). This curve depends on the crust thermal conductivity, crust thickness, distribution of crustal heating sources, and on the neutrino cooling of neutron star core (Rutledge et al. 2002; Cackett et al. 2006).

There are two complementary aspects of observations of cooling of persistent SXTs. On the one hand, we need a theory to understand this phenomenon. On the other hand, observational data yield constraints on theoretical models. Very recently, simulations of thermal relaxation of KS 1731-260 were performed along these lines by Shternin et al. (2007). Increasing in number and precision, observations of cooling curves in persistent SXTs will hopefully be a promising testing ground for the theories of 
deep crustal heating, including pycnonuclear fusion and other physical processes in neutron stars.

Acknowledgements. We are very grateful to D. G. Yakovlev for a critical reading of the manuscript and for helpful remarks. This work was supported in part by the KBN grant 5 P03D 02020 and the MNiSW grant N20300632/0450.

\section{References}

Bildsten, L., \& Chakrabarty, D. 2001, ApJ, 557, 292

Bisnovatyi-Kogan, G. S., \& Chechetkin, V. M. 1979, Uspekhi Fiz. Nauk, 127, 263 (English translation: Sov. Phys. Uspekhi 22, 89)

Brown, E. F., Bildsten, L., \& Rutledge, R. E. 1998, ApJ, 504, L95

Cackett, E. M., Wijnands, R., Linares, M., et al. 2006, MNRAS, 372, 479

Campana, S., Stella, L., Gastaldello, F., et al. 2002, ApJ, 575, L15
Chen, W., Shrader, C. R., \& Livio, M. 1997, ApJ, 491, 312

Colpi, M., Geppert, U., Page, D., \& Possenti, A. 2001, ApJ, 548, L175

Gupta, S., Brown, E. F., Schatz, H., Möller, P., \& Kratz, K.-L. 2007, ApJ, 662, 1188

Haensel, P., \& Zdunik, J. L. 1990, A\&A, 227, 431

Haensel, P., \& Zdunik, J. L. 2003, A\&A, 404, L33

Levenfish, K. P., \& Haensel, P. 2007, Ap\&SS, 308, 457

Rutledge, R. E., Bildsten, L., Brown, E. F., et al. 2002, ApJ, 580, 413

Sato, K. 1979, Prog. Theor. Phys., 62, 957

Schatz, H., Aprahamian, A., Barnard, V., et al. 2001, Phys. Rev. Lett., 86, 3471

Schatz, H., Bildsten, L., \& Cumming, A. 2003, ApJ, 583, L87

Shternin, P. S., Yakovlev, D. G., Haensel, P., \& Potekhin, A. Y. 2007, MNRAS, 382, L43

Vartanyan, Yu. L., \& Ovakimova, N. K. 1976, Soobtcheniya Byurakanskoi Observatorii, 49, 87

Yakovlev, D. G., Levenfish, K. P., \& Haensel, P. 2003, A\&A, 407, 265

Yakovlev, D. G., Levenfish, K. P., Potekhin, A. Y., et al. 2004, A\&A, 417, 169

Yakovlev, D. G., Gasques, L., \& Wiescher, M. 2006, MNRAS, 371, 1322 
P. Haensel and J. L. Zdunik: Crustal heating in accreting neutron stars, Online Material $p 1$

\section{Appendix A: Tables}

In the tables we present the chains of non-equilibrium processes in the crust of an accreting neutron stars, assuming that the X-ray ashes consist of pure ${ }^{56} \mathrm{Fe}$ or ${ }^{106} \mathrm{Pd}$. The pressure and density at which the reaction takes place are denoted by $P_{j}$ and $\rho_{j}$, respectively. $\Delta \rho / \rho_{j}$ is the relative density jump connected with reaction and $Q_{j}$ is the heat deposited in the matter. $X_{\mathrm{n}}$ is the fraction of free neutrons among nucleons, and $\mu_{\mathrm{e}}$ is the electron chemical potential, both in the layer just above the reaction surface. 
P. Haensel and J. L. Zdunik: Crustal heating in accreting neutron stars, Online Material $p 2$

Table A.1. Non-equilibrium processes in the crust of an accreting neutron stars assuming that the X-ray ashes consist of pure ${ }^{106} \mathrm{Pd}$.

\begin{tabular}{|c|c|c|c|c|c|c|}
\hline $\begin{array}{l}P_{j} \\
\left({\left.\text { dyn } \mathrm{cm}^{-2}\right)}\right.\end{array}$ & $\begin{array}{l}\rho_{j} \\
\left(\mathrm{~g} \mathrm{~cm}^{-3}\right)\end{array}$ & Reactions & $\bar{X}_{\mathrm{n}}$ & $\begin{array}{r}\Delta \rho / \rho_{j} \\
\%\end{array}$ & $\begin{array}{r}\mu_{\mathrm{e}} \\
(\mathrm{MeV})\end{array}$ & $\begin{array}{r}Q_{j} \\
(\mathrm{keV})\end{array}$ \\
\hline $9.235 \times 10^{25}$ & $3.517 \times 10^{08}$ & ${ }^{106} \mathrm{Pd} \rightarrow{ }^{106} \mathrm{Ru}-2 \mathrm{e}^{-}+2 v_{\mathrm{e}}$ & 0 & 4.4 & 2.29 & 22.9 \\
\hline $3.603 \times 10^{27}$ & $5.621 \times 10^{09}$ & ${ }^{106} \mathrm{Ru} \rightarrow{ }^{106} \mathrm{Mo}-2 \mathrm{e}^{-}+2 v_{\mathrm{e}}$ & 0 & 4.6 & 6.34 & 22.7 \\
\hline $2.372 \times 10^{28}$ & $2.413 \times 10^{10}$ & ${ }^{106} \mathrm{Mo} \rightarrow{ }^{106} \mathrm{Zr}-2 \mathrm{e}^{-}+2 v_{\mathrm{e}}$ & 0 & 4.9 & 10.43 & 22.4 \\
\hline $8.581 \times 10^{28}$ & $6.639 \times 10^{10}$ & ${ }^{106} \mathrm{Zr} \rightarrow{ }^{106} \mathrm{Sr}-2 \mathrm{e}^{-}+2 \nu_{\mathrm{e}}$ & 0 & 5.1 & 14.56 & 22.2 \\
\hline $2.283 \times 10^{29}$ & $1.455 \times 10^{11}$ & ${ }^{106} \mathrm{Sr} \rightarrow{ }^{106} \mathrm{Kr}-2 \mathrm{e}^{-}+2 v_{\mathrm{e}}$ & 0 & 5.4 & 18.73 & 22.1 \\
\hline $5.025 \times 10^{29}$ & $2.774 \times 10^{11}$ & ${ }^{106} \mathrm{Kr} \rightarrow{ }^{106} \mathrm{Se}-2 \mathrm{e}^{-}+2 v_{\mathrm{e}}$ & 0 & 5.7 & 22.91 & 22.0 \\
\hline $9.713 \times 10^{29}$ & $4.811 \times 10^{11}$ & ${ }^{106} \mathrm{Se} \rightarrow{ }^{106} \mathrm{Ge}-2 \mathrm{e}^{-}+2 v_{\mathrm{e}}$ & 0 & 6.1 & 27.08 & 22.0 \\
\hline $1.703 \times 10^{30}$ & $7.785 \times 10^{11}$ & ${ }^{106} \mathrm{Ge} \rightarrow{ }^{92} \mathrm{Ni}+14 n-4 \mathrm{e}^{-}+4 v_{\mathrm{e}}$ & 0.13 & 13.2 & 31.22 & 110.8 \\
\hline $1.748 \times 10^{30}$ & $8.989 \times 10^{11}$ & ${ }^{92} \mathrm{Ni} \rightarrow{ }^{86} \mathrm{Fe}+6 \mathrm{n}-2 \mathrm{e}^{-}+2 v_{\mathrm{e}}$ & 0.19 & 6.9 & 31.33 & 53.2 \\
\hline $1.924 \times 10^{30}$ & $1.032 \times 10^{12}$ & ${ }^{86} \mathrm{Fe} \rightarrow{ }^{80} \mathrm{Cr}+6 \mathrm{n}-2 \mathrm{e}^{-}+2 v_{\mathrm{e}}$ & 0.25 & 7.3 & 31.02 & 57.5 \\
\hline $2.135 \times 10^{30}$ & $1.197 \times 10^{12}$ & ${ }^{80} \mathrm{Cr} \rightarrow{ }^{74} \mathrm{Ti}+6 \mathrm{n}-2 \mathrm{e}^{-}+2 v_{\mathrm{e}}$ & 0.30 & 7.7 & 32.76 & 62.1 \\
\hline $2.394 \times 10^{30}$ & $1.403 \times 10^{12}$ & ${ }^{74} \mathrm{Ti} \rightarrow{ }^{68} \mathrm{Ca}+6 \mathrm{n}-2 \mathrm{e}^{-}+2 v_{\mathrm{e}}$ & 0.36 & 8.1 & 33.57 & 67.2 \\
\hline $2.720 \times 10^{30}$ & $1.668 \times 10^{12}$ & ${ }^{68} \mathrm{Ca} \rightarrow{ }^{62} \mathrm{Ar}+6 \mathrm{n}-2 \mathrm{e}^{-}+2 v_{\mathrm{e}}$ & 0.42 & 8.5 & 34.45 & 72.9 \\
\hline $3.145 \times 10^{30}$ & $2.016 \times 10^{12}$ & ${ }^{62} \mathrm{Ar} \rightarrow{ }^{56} \mathrm{~S}+6 \mathrm{n}-2 \mathrm{e}^{-}+2 v_{\mathrm{e}}$ & 0.47 & 9.0 & 35.44 & 79.2 \\
\hline $3.723 \times 10^{30}$ & $2.488 \times 10^{12}$ & ${ }^{56} \mathrm{~S} \rightarrow{ }^{50} \mathrm{Si}+6 \mathrm{n}-2 \mathrm{e}^{-}+2 v_{\mathrm{e}}$ & 0.53 & 9.4 & 36.57 & 86.0 \\
\hline $4.549 \times 10^{30}$ & $3.153 \times 10^{12}$ & ${ }^{50} \mathrm{Si} \rightarrow{ }^{42} \mathrm{Mg}+8 \mathrm{n}-2 \mathrm{e}^{-}+2 v_{\mathrm{e}}$ & 0.61 & 8.8 & 37.86 & 94.5 \\
\hline $4.624 \times 10^{30}$ & $3.472 \times 10^{12}$ & $\begin{array}{l}{ }^{42} \mathrm{Mg} \rightarrow{ }^{36} \mathrm{Ne}+6 \mathrm{n}-2 \mathrm{e}^{-}+2 v_{\mathrm{e}} \\
{ }^{36} \mathrm{Ne}+{ }^{36} \mathrm{Ne} \rightarrow{ }^{72} \mathrm{Ca}\end{array}$ & 0.66 & 10.6 & 37.13 & 268.2 \\
\hline $5.584 \times 10^{30}$ & $4.399 \times 10^{12}$ & ${ }^{72} \mathrm{Ca} \rightarrow{ }^{66} \mathrm{Ar}+6 \mathrm{n}-2 \mathrm{e}^{-}+2 v_{\mathrm{e}}$ & 0.69 & 4.8 & 37.56 & 31.6 \\
\hline $6.883 \times 10^{30}$ & $5.355 \times 10^{12}$ & ${ }^{66} \mathrm{Ar} \rightarrow{ }^{60} \mathrm{~S}+6 \mathrm{n}-2 \mathrm{e}^{-}+2 v_{\mathrm{e}}$ & 0.72 & 4.7 & 39.00 & 33.5 \\
\hline $8.749 \times 10^{30}$ & $6.655 \times 10^{12}$ & ${ }^{60} \mathrm{~S} \rightarrow{ }^{54} \mathrm{Si}+6 \mathrm{n}-2 \mathrm{e}^{-}+2 v_{\mathrm{e}}$ & 0.75 & 4.6 & 40.33 & 35.2 \\
\hline $1.157 \times 10^{31}$ & $8.487 \times 10^{12}$ & $\begin{array}{l}{ }^{54} \mathrm{Si} \rightarrow{ }^{46} \mathrm{Mg}+8 \mathrm{n}-2 \mathrm{e}^{-}+2 v_{\mathrm{e}} \\
{ }^{46} \mathrm{Mg}+{ }^{46} \mathrm{Mg} \rightarrow{ }^{92} \mathrm{Cr}\end{array}$ & 0.79 & 4.0 & 41.84 & 145.3 \\
\hline $1.234 \times 10^{31}$ & $9.242 \times 10^{12}$ & ${ }^{92} \mathrm{Cr} \rightarrow{ }^{86} \mathrm{Ti}+6 \mathrm{n}-2 \mathrm{e}^{-}+2 v_{\mathrm{e}}$ & 0.80 & 2.0 & 40.88 & 11.4 \\
\hline $1.528 \times 10^{31}$ & $1.096 \times 10^{13}$ & ${ }^{86} \mathrm{Ti} \rightarrow{ }^{80} \mathrm{Ca}+6 \mathrm{n}-2 \mathrm{e}^{-}+2 v_{\mathrm{e}}$ & 0.82 & 1.9 & 42.04 & 11.4 \\
\hline $1.933 \times 10^{31}$ & $1.317 \times 10^{13}$ & ${ }^{80} \mathrm{Ca} \rightarrow{ }^{74} \mathrm{Ar}+6 \mathrm{n}-2 \mathrm{e}^{-}+2 v_{\mathrm{e}}$ & 0.83 & 1.8 & 43.31 & 11.2 \\
\hline $2.510 \times 10^{31}$ & $1.609 \times 10^{13}$ & ${ }^{74} \mathrm{Ar} \rightarrow{ }^{68} \mathrm{~S}+6 \mathrm{n}-2 \mathrm{e}^{-}+2 v_{\mathrm{e}}$ & 0.85 & 1.7 & 44.71 & 10.6 \\
\hline $3.363 \times 10^{31}$ & $2.003 \times 10^{13}$ & $\begin{array}{l}{ }^{68} \mathrm{~S} \rightarrow{ }^{62} \mathrm{Si}+6 \mathrm{n}-2 \mathrm{e}^{-}+2 v_{\mathrm{e}} \\
{ }^{62} \mathrm{Si}+{ }^{62} \mathrm{Si} \rightarrow{ }^{124} \mathrm{Ni}\end{array}$ & 0.86 & 1.7 & 46.26 & 70.5 \\
\hline $4.588 \times 10^{31}$ & $2.520 \times 10^{13}$ & ${ }^{124} \mathrm{Ni} \rightarrow{ }^{120} \mathrm{Fe}+4 \mathrm{n}-2 \mathrm{e}^{-}+2 v_{\mathrm{e}}$ & 0.87 & 0.8 & 47.78 & 3.0 \\
\hline $5.994 \times 10^{31}$ & $3.044 \times 10^{13}$ & ${ }^{120} \mathrm{Fe} \rightarrow{ }^{118} \mathrm{Cr}+2 \mathrm{n}-2 \mathrm{e}^{-}+2 v_{\mathrm{e}}$ & 0.88 & 0.9 & 49.65 & 2.7 \\
\hline $8.408 \times 10^{31}$ & $3.844 \times 10^{13}$ & ${ }^{118} \mathrm{Cr} \rightarrow{ }^{116} \mathrm{Ti}+2 \mathrm{n}-2 \mathrm{e}^{-}+2 v_{\mathrm{e}}$ & 0.88 & 0.8 & 52.28 & 2.5 \\
\hline
\end{tabular}

Table A.2. Nuclear processes and released heat in the inner crust, assuming initial ashes of pure ${ }^{106} \mathrm{Pd}$ (i.e. as in Table A.1) but suppressing pycnonuclear fusion until $Z=Z_{\min }=4$. The network of reactions below pressure $P=4.624 \times 10^{30} \mathrm{dyn} \mathrm{cm}^{-2}$ is the same as in the Table A.1.

\begin{tabular}{lllrrrr}
\hline \hline $\begin{array}{l}P_{j} \\
(\text { dyn cm }\end{array}$ & $\begin{array}{l}\left.\rho_{j}\right) \\
\left(\mathrm{g} \mathrm{cm}^{-3}\right)\end{array}$ & Reactions & $X_{\mathrm{n}}$ & $\begin{array}{r}\Delta \rho / \rho_{j} \\
\%\end{array}$ & $\begin{array}{r}\mu_{\mathrm{e}} \\
(\mathrm{MeV})\end{array}$ & $\begin{array}{r}Q_{j} \\
(\mathrm{keV})\end{array}$ \\
\hline $4.624 \times 10^{30}$ & $3.472 \times 10^{12}$ & ${ }^{42} \mathrm{Mg} \rightarrow{ }^{36} \mathrm{Ne}+6 \mathrm{n}-2 \mathrm{e}^{-}+2 v_{\mathrm{e}}$ & 0.66 & 9.9 & 37.13 & 76.0 \\
$6.253 \times 10^{30}$ & $4.745 \times 10^{12}$ & ${ }^{36} \mathrm{Ne} \rightarrow{ }^{30} \mathrm{O}+6 \mathrm{n}-2 \mathrm{e}^{-}+2 v_{\mathrm{e}}$ & 0.72 & 9.9 & 38.79 & 84.2 \\
$9.323 \times 10^{30}$ & $6.937 \times 10^{12}$ & ${ }^{30} \mathrm{O} \rightarrow{ }^{24} \mathrm{C}+6 \mathrm{n}-2 \mathrm{e}^{-}+2 v_{\mathrm{e}}$ & 0.77 & 9.4 & 40.89 & 91.8 \\
$1.615 \times 10^{31}$ & $1.119 \times 10^{12}$ & ${ }^{24} \mathrm{C} \rightarrow{ }^{18} \mathrm{Be}+6 \mathrm{n}-2 \mathrm{e}^{-}+2 v_{\mathrm{e}}$ & 0.84 & 9.2 & 43.59 & 94.4 \\
\hline $3.500 \times 10^{31}$ & $2.071 \times 10^{13}$ & ${ }^{18} \mathrm{Be} \rightarrow{ }^{15} \mathrm{Li}+3 \mathrm{n}-1 \mathrm{e}^{-}+1 v_{\mathrm{e}}$ & & & & \\
& & ${ }^{15} \mathrm{Li}+{ }^{15} \mathrm{Li} \rightarrow{ }^{28} \mathrm{C}+2 n$ & 0.88 & 2.3 & 46.78 & 152.8 \\
\hline $6.339 \times 10^{31}$ & $3.166 \times 10^{13}$ & ${ }^{28} \mathrm{C} \rightarrow{ }^{27} \mathrm{~B}+1 \mathrm{n}-1 \mathrm{e}^{-}+1 v_{\mathrm{e}}$ & 0.89 & 1.9 & 48.98 & 8.7 \\
\hline
\end{tabular}


P. Haensel and J. L. Zdunik: Crustal heating in accreting neutron stars, Online Material $p 3$

Table A.3. Non-equilibrium processes in the crust of an accreting neutron stars assuming that the X-ray ashes consist of pure ${ }^{56} \mathrm{Fe}$.

\begin{tabular}{|c|c|c|c|c|c|c|}
\hline $\begin{array}{l}P \\
\left(\text { dyn } \mathrm{cm}^{-2}\right)\end{array}$ & $\begin{array}{l}\rho \\
\left(\mathrm{g} \mathrm{cm}^{-3}\right)\end{array}$ & Reactions & $\overline{X_{\mathrm{n}}}$ & $\overline{\Delta \Delta \rho / \rho}$ & $\mu_{\mathrm{e}}$ & $\begin{array}{l}q \\
(\mathrm{keV})\end{array}$ \\
\hline $7.23 \times 10^{26}$ & $1.49 \times 10^{9}$ & ${ }^{56} \mathrm{Fe} \rightarrow{ }^{56} \mathrm{Cr}-2 \mathrm{e}^{-}+2 v_{\mathrm{e}}$ & 0 & 0.08 & 4.08 & 40.7 \\
\hline $9.57 \times 10^{27}$ & $1.11 \times 10^{10}$ & ${ }^{56} \mathrm{Cr} \rightarrow{ }^{56} \mathrm{Ti}-2 \mathrm{e}^{-}+2 v_{\mathrm{e}}$ & 0 & 0.09 & 8.18 & 35.8 \\
\hline $1.15 \times 10^{29}$ & $7.85 \times 10^{10}$ & ${ }^{56} \mathrm{Ti} \rightarrow{ }^{56} \mathrm{Ca}-2 \mathrm{e}^{-}+2 v_{\mathrm{e}}$ & 0 & 0.10 & 15.64 & 47.3 \\
\hline $4.75 \times 10^{29}$ & $2.50 \times 10^{11}$ & ${ }^{56} \mathrm{Ca} \rightarrow{ }^{56} \mathrm{Ar}-2 \mathrm{e}^{-}+2 v_{\mathrm{e}}$ & 0 & 0.11 & 22.48 & 46.1 \\
\hline $1.36 \times 10^{30}$ & $6.11 \times 10^{11}$ & ${ }^{56} \mathrm{Ar} \rightarrow{ }^{52} \mathrm{~S}+4 \mathrm{n}-2 \mathrm{e}^{-}+2 v_{\mathrm{e}}$ & 0 & 0.12 & 29.38 & 59.8 \\
\hline $1.980 \times 10^{30}$ & $9.075 \times 10^{11}$ & ${ }^{52} \mathrm{~S} \rightarrow{ }^{46} \mathrm{Si}+6 \mathrm{n}-2 \mathrm{e}^{-}+2 v_{\mathrm{e}}$ & 0.07 & 0.13 & 32.27 & 128.0 \\
\hline $2.253 \times 10^{30}$ & $1.131 \times 10^{12}$ & ${ }^{46} \mathrm{Si} \rightarrow{ }^{40} \mathrm{Mg}+6 \mathrm{n}-2 \mathrm{e}^{-}+2 v_{\mathrm{e}}$ & 0.18 & 0.14 & 32.22 & 143.5 \\
\hline $2.637 \times 10^{30}$ & $1.455 \times 10^{12}$ & $\begin{array}{l}{ }^{40} \mathrm{Mg} \rightarrow{ }^{34} \mathrm{Ne}+6 \mathrm{n}-2 \mathrm{e}^{-}+2 v_{\mathrm{e}} \\
{ }^{34} \mathrm{Ne}+{ }^{34} \mathrm{Ne} \rightarrow{ }^{68} \mathrm{Ca}\end{array}$ & 0.39 & 0.17 & 34.34 & 507.9 \\
\hline $2.771 \times 10^{30}$ & $1.766 \times 10^{12}$ & ${ }^{68} \mathrm{Ca} \rightarrow{ }^{62} \mathrm{Ar}+6 \mathrm{n}-2 \mathrm{e}^{-}+2 v_{\mathrm{e}}$ & 0.45 & 0.8 & 34.47 & 65.8 \\
\hline $3.216 \times 10^{30}$ & $2.134 \times 10^{12}$ & ${ }^{62} \mathrm{Ar} \rightarrow{ }^{56} \mathrm{~S}+6 \mathrm{n}-2 \mathrm{e}^{-}+2 v_{\mathrm{e}}$ & 0.45 & 0.09 & 35.47 & 71.6 \\
\hline $3.825 \times 10^{30}$ & $2.634 \times 10^{12}$ & ${ }^{56} \mathrm{~S} \rightarrow{ }^{50} \mathrm{Si}+6 \mathrm{n}-2 \mathrm{e}^{-}+2 v_{\mathrm{e}}$ & 0.50 & 0.09 & 36.59 & 77.9 \\
\hline $4.699 \times 10^{30}$ & $3.338 \times 10^{12}$ & ${ }^{50} \mathrm{Si} \rightarrow{ }^{44} \mathrm{Mg}+6 \mathrm{n}-2 \mathrm{e}^{-}+2 v_{\mathrm{e}}$ & 0.55 & 0.09 & 37.89 & 84.6 \\
\hline $6.043 \times 10^{30}$ & $4.379 \times 10^{12}$ & $\begin{array}{l}{ }^{44} \mathrm{Mg} \rightarrow{ }^{36} \mathrm{Ne}+8 \mathrm{n}-2 \mathrm{e}^{-}+2 v_{\mathrm{e}} \\
{ }^{36} \mathrm{Ne}+{ }^{36} \mathrm{Ne} \rightarrow{ }^{72} \mathrm{Ca} \\
{ }^{72} \mathrm{Ca} \rightarrow{ }^{66} \mathrm{Ar}+6 \mathrm{n}-2 \mathrm{e}^{-}+2 v_{\mathrm{e}}\end{array}$ & 0.61 & 0.14 & 39.41 & 308.8 \\
\hline $7.233 \times 10^{30}$ & $5.839 \times 10^{12}$ & ${ }^{66} \mathrm{Ar} \rightarrow{ }^{60} \mathrm{~S}+6 \mathrm{n}-2 \mathrm{e}^{-}+2 v_{\mathrm{e}}$ & 0.70 & 0.04 & 39.01 & 29.5 \\
\hline $9.238 \times 10^{30}$ & $7.041 \times 10^{12}$ & ${ }^{60} \mathrm{~S} \rightarrow{ }^{54} \mathrm{Si}+6 \mathrm{n}-2 \mathrm{e}^{-}+2 v_{\mathrm{e}}$ & 0.73 & 0.04 & 40.34 & 31.0 \\
\hline $1.228 \times 10^{31}$ & $8.980 \times 10^{12}$ & $\begin{array}{l}{ }^{54} \mathrm{Si} \rightarrow{ }^{48} \mathrm{Mg}+6 \mathrm{n}-2 \mathrm{e}^{-}+2 v_{\mathrm{e}} \\
{ }^{48} \mathrm{Mg}+{ }^{48} \mathrm{Mg} \rightarrow{ }^{96} \mathrm{Cr} \\
{ }^{96} \mathrm{Cr} \rightarrow{ }^{94} \mathrm{Cr}+2 \mathrm{n}\end{array}$ & 0.80 & 0.04 & 41.86 & 135.1 \\
\hline $1.463 \times 10^{31}$ & $1.057 \times 10^{13}$ & ${ }^{94} \mathrm{Cr} \rightarrow{ }^{88} \mathrm{Ti}+6 \mathrm{n}-2 \mathrm{e}^{-}+2 v_{\mathrm{e}}$ & 0.81 & 0.02 & 41.99 & 11.5 \\
\hline $1.816 \times 10^{31}$ & $1.254 \times 10^{13}$ & ${ }^{88} \mathrm{Ti} \rightarrow{ }^{82} \mathrm{Ca}+6 \mathrm{n}-2 \mathrm{e}^{-}+2 v_{\mathrm{e}}$ & 0.82 & 0.02 & 43.18 & 11.3 \\
\hline $2.304 \times 10^{31}$ & $1.506 \times 10^{13}$ & ${ }^{82} \mathrm{Ca} \rightarrow{ }^{76} \mathrm{Ar}+6 \mathrm{n}-2 \mathrm{e}^{-}+2 v_{\mathrm{e}}$ & 0.84 & 0.02 & 44.48 & 10.9 \\
\hline $2.998 \times 10^{31}$ & $1.838 \times 10^{13}$ & ${ }^{76} \mathrm{Ar} \rightarrow{ }^{70} \mathrm{~S}+6 \mathrm{n}-2 \mathrm{e}^{-}+2 v_{\mathrm{e}}$ & 0.85 & 0.02 & 45.91 & 10.0 \\
\hline $4.028 \times 10^{31}$ & $2.287 \times 10^{13}$ & $\begin{array}{l}{ }^{70} \mathrm{~S} \rightarrow{ }^{64} \mathrm{Si}+6 \mathrm{n}-2 \mathrm{e}^{-}+2 v_{\mathrm{e}} \\
{ }^{64} \mathrm{Si}+{ }^{64} \mathrm{Si} \rightarrow \rightarrow^{128} \mathrm{Ni} \\
{ }^{128} \mathrm{Ni} \rightarrow{ }^{126} \mathrm{Ni}+2 \mathrm{n}\end{array}$ & 0.87 & 0.01 & 47.48 & 67.3 \\
\hline $5.278 \times 10^{31}$ & $2.784 \times 10^{13}$ & ${ }^{126} \mathrm{Ni} \rightarrow{ }^{124} \mathrm{Fe}+2 \mathrm{n}-2 \mathrm{e}^{-}+2 v_{\mathrm{e}}$ & 0.88 & 0.01 & 48.50 & 2.5 \\
\hline $7.311 \times 10^{31}$ & $3.493 \times 10^{13}$ & ${ }^{124} \mathrm{Fe} \rightarrow{ }^{122} \mathrm{Cr}+2 \mathrm{n}-2 \mathrm{e}^{-}+2 v_{\mathrm{e}}$ & 0.89 & 0.01 & 51.05 & 2.4 \\
\hline
\end{tabular}

Table A.4. Nuclear processes and released heat in the inner crust, assuming initial ashes of pure ${ }^{56} \mathrm{Fe}$ (i.e. as in Table A.3) but suppressing pycnonuclear fusion until $Z=Z_{\min }=4$. The network of reactions below pressure $P=2.637 \times 10^{30}$ dyn cm $\mathrm{cm}^{-2}$ is the same as in Table A.3.

\begin{tabular}{lllrrrr}
\hline \hline $\left.\begin{array}{l}P \\
(\text { dyn cm }\end{array}{ }^{-2}\right)$ & $\begin{array}{l}\rho \\
\left(\mathrm{g} \mathrm{cm}^{-3}\right)\end{array}$ & Reactions & $X_{\mathrm{n}}$ & $\begin{array}{r}\Delta \rho / \rho \\
\%\end{array}$ & $\begin{array}{r}\mu_{\mathrm{e}} \\
(\mathrm{MeV})\end{array}$ & $\begin{array}{r}q \\
(\mathrm{keV})\end{array}$ \\
\hline $2.637 \times 10^{30}$ & $1.455 \times 10^{12}$ & ${ }^{40} \mathrm{Mg} \rightarrow{ }^{34} \mathrm{Ne}+6 \mathrm{n}-2 \mathrm{e}^{-}+2 v_{\mathrm{e}}$ & 0.40 & 16.0 & 34.45 & 161.4 \\
$3.227 \times 10^{30}$ & $1.961 \times 10^{12}$ & ${ }^{34} \mathrm{Ne} \rightarrow{ }^{28} \mathrm{O}+6 \mathrm{n}-2 \mathrm{e}^{-}+2 v_{\mathrm{e}}$ & 0.50 & 17.9 & 35.71 & 183.4 \\
$4.254 \times 10^{30}$ & $2.831 \times 10^{12}$ & ${ }^{28} \mathrm{O} \rightarrow{ }^{22} \mathrm{C}+6 \mathrm{n}-2 \mathrm{e}^{-}+2 v_{\mathrm{e}}$ & 0.61 & 19.4 & 37.49 & 209.8 \\
$6.392 \times 10^{30}$ & $4.541 \times 10^{12}$ & ${ }^{22} \mathrm{C} \rightarrow{ }^{16} \mathrm{Be}+6 \mathrm{n}-2 \mathrm{e}^{-}+2 v_{\mathrm{e}}$ & 0.72 & 20.0 & 39.89 & 238.2 \\
\hline $1.216 \times 10^{31}$ & $8.617 \times 10^{12}$ & ${ }^{16} \mathrm{Be} \rightarrow{ }^{13} \mathrm{Li}+4 \mathrm{n}-1 \mathrm{e}^{-}+1 v_{\mathrm{e}}$ & & & & \\
& & ${ }^{13} \mathrm{Li}+{ }^{13} \mathrm{Li} \rightarrow{ }^{26} \mathrm{C}$ & & & \\
& & ${ }^{26} \mathrm{C} \rightarrow{ }^{24} \mathrm{C}+2 \mathrm{n}$ & & & \\
& & ${ }^{24} \mathrm{C} \rightarrow{ }^{18} \mathrm{Be}+6 \mathrm{n}-2 \mathrm{e}^{-}+2 v_{\mathrm{e}}$ & 0.85 & 7.4 & 43.64 & 84.1 \\
\hline $1.727 \times 10^{31}$ & $1.186 \times 10^{13}$ & & & & \\
\hline $3.798 \times 10^{31}$ & $2.200 \times 10^{12}$ & ${ }^{18} \mathrm{Be} \rightarrow{ }^{17} \mathrm{Li}+1 \mathrm{n}-1 \mathrm{e}^{-}+1 v_{\mathrm{e}}$ & & & & \\
& ${ }^{17} \mathrm{Li}+{ }^{17} \mathrm{Li} \rightarrow{ }^{34} \mathrm{C}$ & 0.88 & 0.028 & 46.86 & 142.0 \\
\hline & ${ }^{34} \mathrm{C} \rightarrow{ }^{30} \mathrm{C}+4 \mathrm{n}$ & & & &
\end{tabular}

